\title{
Effects of spatial parameters on the perceptual organization of a bistable motion display
}

\author{
ALLAN J. PANTLE and J. TIMOTHY PETERSIK \\ Miami University, Oxford, Ohio 45056
}

\begin{abstract}
In a dynamic visual display which consisted of two alternating frames, each with three line elements, the individual elements which possessed a high degree of spatial correspondence from one frame to the next were paired perceptually and moved about locally. Any remaining elements moved independently and followed different movement rules. When there was no high degree of spatial correspondence of any elements across frames, or when the interval of time between frames was long, the elements behaved as a perceptual unit and moved about as a group. The local pairings and movements appeared to be mediated by a process with different functional characteristics from those of the process which mediated the wholistic movement of the elements as a group.
\end{abstract}

The purpose of the present experiments was to study the nature of the perceptual organizations imposed by our visual system on a dynamic display that was employed by Pantle and Picciano (1976) and resembled one described by Ternus (1938). With the display, competing sensations of stroboscopic movement are produced by a cyclic alternation of two stimulus frames. Frame 1 contains three stimulus elements (A, B, C) arranged in a horizontal row on a blank background. Frame 2 contains three identical elements $(D, E, F)$, also arranged horizontally but shifted to the right such that the positions of $D$ and $E$ of Frame 2 overlap those of B and C, respectively, of Frame 1 (see Figure 1). When stimulus conditions are appropriately chosen, the spatiotemporal display gives rise to a bistable percept: Either an observer perceives a group of three elements moving back and forth as a whole (group movement) or he perceives the overlapping elements of each frame as stationary and a third element moving back and forth from one end of the display to the other (element movement).

The rationale for the two specific objectives of our present research with the element-group movement display (hereafter, EG display) is as follows:

(1) As Petersik and Pantle (1979) elaborated in detail, stimulus parameters of the EG display can be manipulated so as to favor either the element or group movement sensation. The values of the parameters which favor element movement were shown to be the same as those required for an observer to segregate and perceive the apparent movement of corre-

This research was supported in part by Air Force Contract F33615-76-C-5006, sponsored by the Aerospace Medical Research Laboratory, Wright-Patterson Air Force Base, Ohio 45433. We thank Peggy Johnston and Ken Hicks for their help. J. T. Petersik is now at Southeast Missouri State University, Department of Psychology, Cape Girardeau, Missouri 63701. lated areas in alternating, Julesz-type, random-element' patterns. For this reason, the process mediating the element movement sensation (termed the $\varepsilon$-process by Petersik and Pantle, 1979) was identified with the process responsible for the segregation and perceived movement of subjective figures in random-element displays (termed the short-range process by Braddick, 1974). The basis for the operation of the short-range process is the physical identity of a subset of elements or points in successive frames of a display sequence (Anstis, 1970; Bell \& Lappin, 1973; Braddick, 1973, 1974; Julesz, 1971). In order that the subset of elements or points be linked across frames and emerge

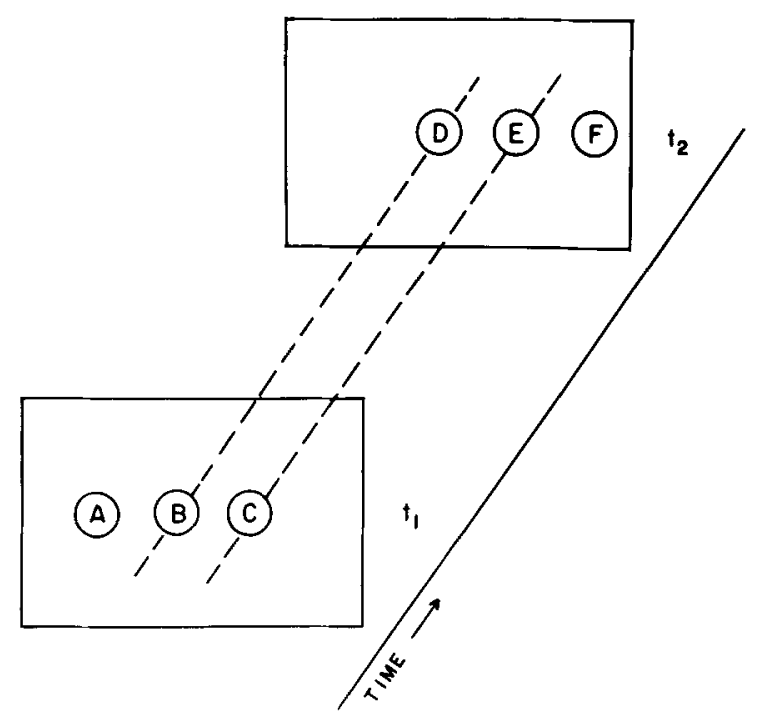

Figure 1. A schematic representation of the spatiotemporal characteristics of the display used to produce bistable movement sensations. 
as a subjective figure, the shift in the location of the subset from one frame to the next cannot be too large

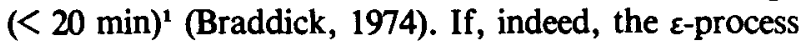
is identical in its functional characteristics to the short-range process, we reasoned that the strength of the element movement sensation might depend upon the extent to which parts of the separate frames of the EG display were in exact, or nearly exact, correspondence. It would follow that spatial perturbations (rotations or shifts) of the overlapping elements of one frame (say, D and E in Figure 1) of the display would weaken the element-movement sensation.

Just as the element movement sensation has limiting conditions which are like those for the segregation and perception of movement of correlated areas in random-dot patterns, the group movement sensation is favored by the same stimulus conditions that are required to obtain stroboscopic movement of texture clusters (Pantle, 1973; Ramachandran, Madhusudhan Rao, \& Vidyasagar, 1973). In those studies, the alternation of a pair of frames, each of which contained a cluster of elements defined by a global form cue, caused an observer to perceive the cluster to move back and forth as a whole. The cluster movement sensation occurred despite the fact that the internal arrangement of the elements of the cluster did not remain the same from one frame to the next. The group movement sensation may simply be a specific instance of the cluster movement sensation (mediated by the $\gamma$-process of Petersik \& Pantle, 1979) in which the three lines of the EG display are processed as a cluster whose average brightness differs from that of the surrounding blank background. If so, small systematic perturbations of the individual elements of one frame of the display should not affect the strength of the group movement sensation.

In summary, spatial perturbations ought to weaken the element movement sensation, but not the group movement sensation, and, therefore, the percentage of group movement sensations ought to increase as the degree of the spatial perturbations is increased.

(2) With the spatial separation of $1 \mathrm{deg}$ or more between adjacent elements typically used in the EG display (Braddick \& Adlard, 1978; Pantle \& Picciano, 1976; Petersik \& Pantle, 1979), only two local pairings are possible between the elements of successive frames, namely the overlapping elements. However, if the spacing between all elements is reduced, local pairings among all three elements of the successive frames may be possible, and the element movement sensation may be abolished in favor of one in which all three elements move together locally.

\section{EXPERIMENT 1}

In all our experiments, we used lines as elements. In the first experiment, we introduced small system- atic perturbations in the orientation of the two line elements of one frame (elements which corresponded to $D$ and $E$ of the frame at time $t_{2}$ in Figure 1) which overlapped the two line elements ( $B$ and $C$ ) of the other frame. If the processes underlying the element and group movement sensations are differentially sensitive to the tilts of the line elements, then one would expect them to alter the proportion of element and group movement responses.

\section{Method}

Subjects. The observers were eight undergraduate psychology students, six of whom served in partial fulfillment of course requirements. Two of the subjects were experienced psychophysical observers, but all subjects were naive with respect to the purpose of the experiment.

Stimuli and Apparatus. The stimuli were similar to those shown in Figure 1, with the exception that the stimuli were light green lines which appeared on a darker background. Each stimulus frame was presented on a CRT screen coated with a rapid decay phosphor (P31) by a PDP-11/10 computer. The viewing distance was $104 \mathrm{~cm}$, and at this distance each line subtended $3 \mathrm{~min} 40 \mathrm{sec}$ of visual angle in width and $3 \mathrm{deg} 8 \mathrm{~min}$ in height. The separation between adjacent lines was $1 \mathrm{deg}$. Stimulus frames were viewed with ambient room illumination, and the resultant luminance of the CRT screen was $.38 \mathrm{~cd} / \mathrm{m}^{2}$. Against the background of the CRT screen, each of the stimulus lines generated by the computer had a luminance of $22.9 \mathrm{~cd} / \mathrm{m}^{2}$. The oscilloscope screen subtended $11 \mathrm{deg} 45 \mathrm{~min}$ vertically and $15 \mathrm{deg} 15 \mathrm{~min}$ horizontally.

Procedure. Each subject served in five experimental sessions, and in each session he viewed two randomized blocks of 20 trials. There was one trial in each block for each combination of the four displays described below and five intervals between frames (ISIs), On each trial, a subject viewed the alternating frames of a display for $3 \mathrm{sec}$. Each frame in the sequence had a duration of $200 \mathrm{msec}$. For each trial, the subject's task was to report whether he saw element or group movement.

\section{Results}

Figure $2 \mathrm{~A}$ is a schematic representation of the control display (tilt perturbation $=0 \mathrm{deg}$ ) used in the present experiment. In the control display, all lines were vertical, the interelement spacing $(X)$ was $1 \mathrm{deg}$, and lines $\mathrm{D}$ and $\mathrm{E}$ (letter denotations as defined in Figure 1) of the frame at time $t_{2}$ exactly overlapped the positions of lines $B$ and $C$ of the frame at time $t_{1}$. However, the computer was programmed such that the lines $D$ and $E$ in the frame at time $t_{2}$ could be tilted about their centers to 3,6 , or $8 \mathrm{deg}$ clockwise from vertical to produce three different experimental displays.

Along with the control display, the three experimental displays were used to generate four functions over five values of ISI $(0,17,33,50$, and $67 \mathrm{msec})$. Thus, each curve in Figure 3 shows, for a single tilt, the percentage of group movement responses as a function of ISI. ${ }^{2}$ Each point on a curve is based upon 80 responses, 10 for each of eight subjects. As can be seen in Figure 3, the subjects reported a greater percentage of group movement sensations with larger tilts. In general, the curve for each suc- 
cessively larger tilt lies above the curve for a lesser tilt. The increase in the percentage of group movement responses with greater tilts is not large at the 67-msec ISI because subjects had already reported group movement nearly $100 \%$ of the time at the 67-msec ISI even with the control display. A repeated measures analysis of variance of the percentage of group movement responses verified the significant main effect of tilt $[\mathrm{F}(3,21)=31.4, \mathrm{p}<.0001]$.

In addition, as shown in Figure 3, for each tilt an increase of ISI produced an increase in the percentage of group movement responses. At short ISIs the element movement sensation was dominant, while at longer ISIs the group movement sensation was dominant. The main effect of ISI was significant $[F(4,28)=89.8, p<.0001]$, replicating the effect first reported by Pantle and Picciano (1976).

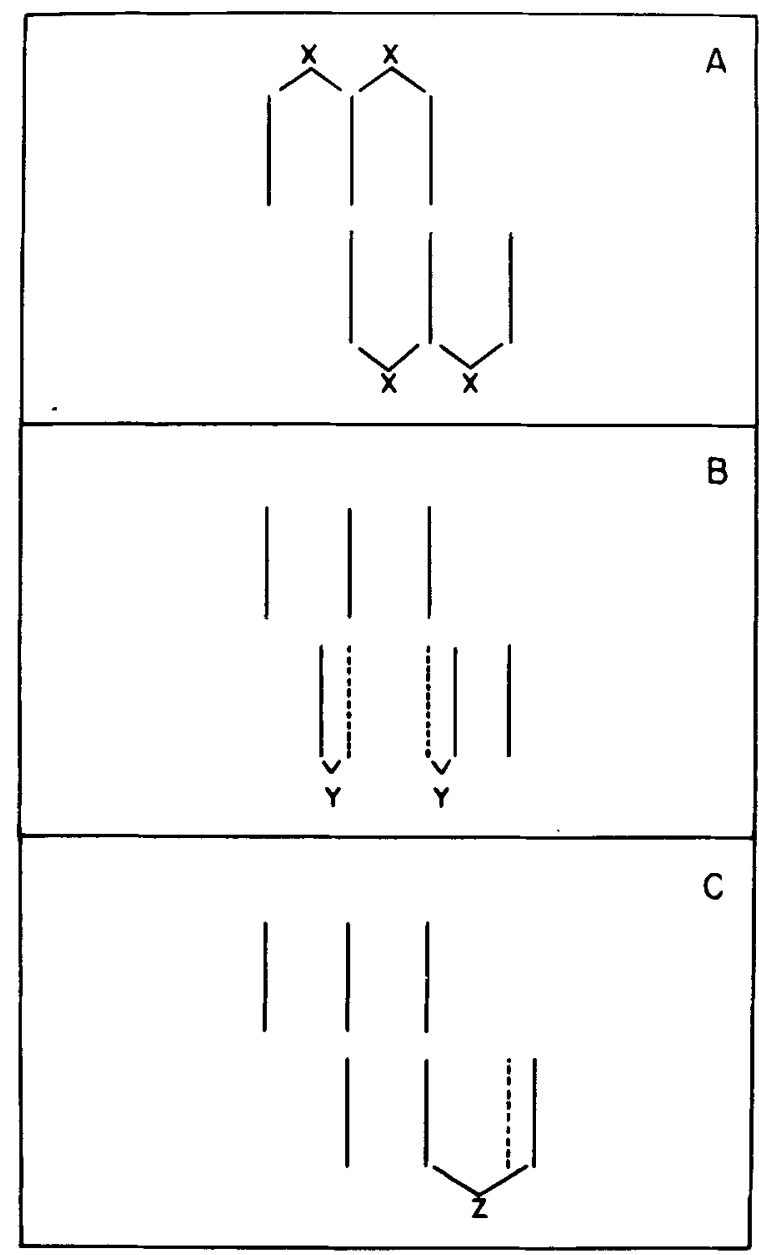

Figure 2. A schematic representation of the control display (A) and of the variables of interelement spacing $(X)$, position perturbation of the overlapping lines ( $Y$ ), and position perturbation of the end line $(Z)$.

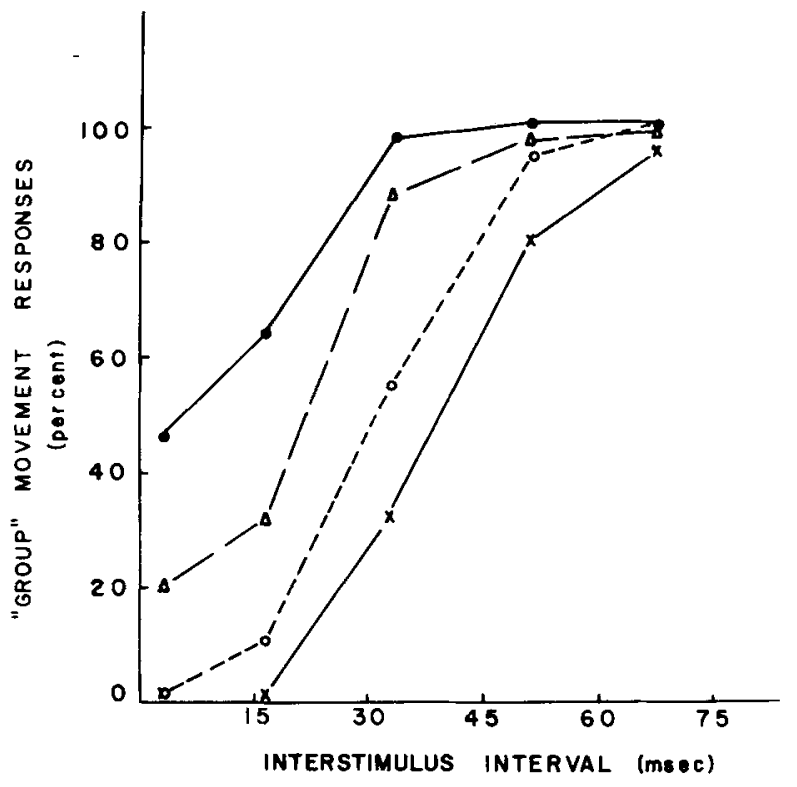

Figure 3. Percentage of group movement responses as a function of the duration of the interstimulus interval with tilt perturbation magnitude of the overlapping lines as a parameter. Tilt perturbation of 0 deg (control data), $\times$ s; $3 \mathrm{deg}$, Os; $6 \mathrm{deg}, \Delta s$; and $8 \mathrm{deg}$, os.

\section{EXPERIMENT 2}

To produce the control display of Experiment 2, two stimulus frames, which were identical to those described in the previous experiment and which contained no tilt perturbations (see Figure 2A), were presented in alternate succession. The other experimental displays were designed to investigate the effects of two factors on the relative proportion of element and group movement sensations: (1) position perturbations and (2) interelement spacing, both described below. Although data with all the displays were collected in the same sessions, it is easiest to think of the displays as belonging to three separate subexperiments. We have arranged the description of the displays and the results accordingly. Common features of the method used with all of the displays are described first.

\section{Method}

Subjects. Six undergraduate psychology students participated in the experiment, four of them to satisfy course requirements and two as volunteers. The two volunteers were experienced psychophysical observers and had participated in Experiment 1. All subjects were naive with respect to the purpose of Experiment 2.

Procedure. Experiment 2 was conducted in the same way as Experiment 1 with a few exceptions. A subject served in 10 experimental sessions, and in each session he viewed a randomized block of $\mathbf{4 0}$ trials in which he made one judgment at each of five ISIs for the control display and each of seven experimental displays. 


\section{Results}

Position perturbations of overlapping lines. The two stimulus frames shown in Figure $2 \mathrm{~B}$ represent a condition in which the location of the "overlapping" lines of one stimulus frame have been shifted laterally by a distance, $Y$, relative to their locations in the control condition (represented by the dashed lines in Figure 2B). (By definition, the shift of the overlapping lines in the control condition was $0 \mathrm{deg}$.) The computer was programmed so that the lateral shift $\mathbf{Y}$ could be 4,8 , or 16 min of visual angle.

Along with the control display, the three experimental displays were used to generate four functions over the five values of ISI. Each curve in Figure 4 shows, for a single position perturbation $Y$, the mean percentage of group movement responses as a function of ISI. As can be seen in Figure 4, the larger the position perturbation, the greater was the percentage of group movement responses. The curve for each larger perturbation $\mathrm{Y}$ lies above the curve for a smaller perturbation. At long ISIs, the change in group movement responses was small because a "ceiling" of $100 \%$ group movement responses was approached for all degrees of perturbation. A repeated measures analysis of variance of the percentage of group movement responses verified the significant main effect of position perturbation (magnitude of $Y$ ) $[F(3,15)=34.4$, $\mathrm{p}<.0001$ ]. The main effect of ISI, namely that more group movement was seen with longer ISIs, was also significant $[F(4,20)=46.3, p<.0001]$.

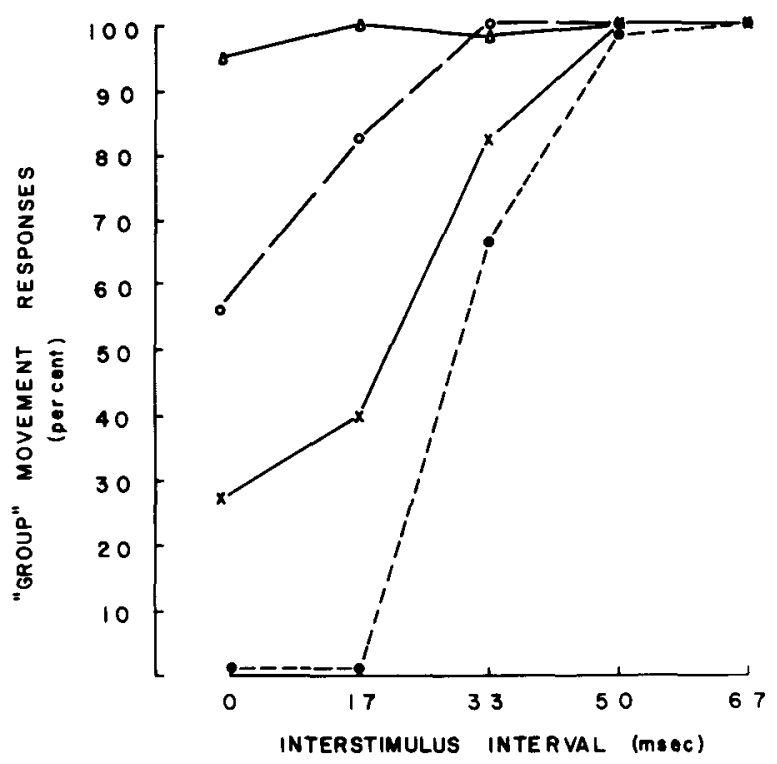

Figure 4. Percentage of group movement responses as a function of the duration of the interstimulus interval with position perturbation magnitude of the overlapping lines as a parameter. Position perturbation (Y) of 0 min (control data), 9 ; 4 min, $\times$ s; $8 \mathrm{~min}$, Os; and $16 \mathrm{~min}, \Delta \mathrm{s}$.

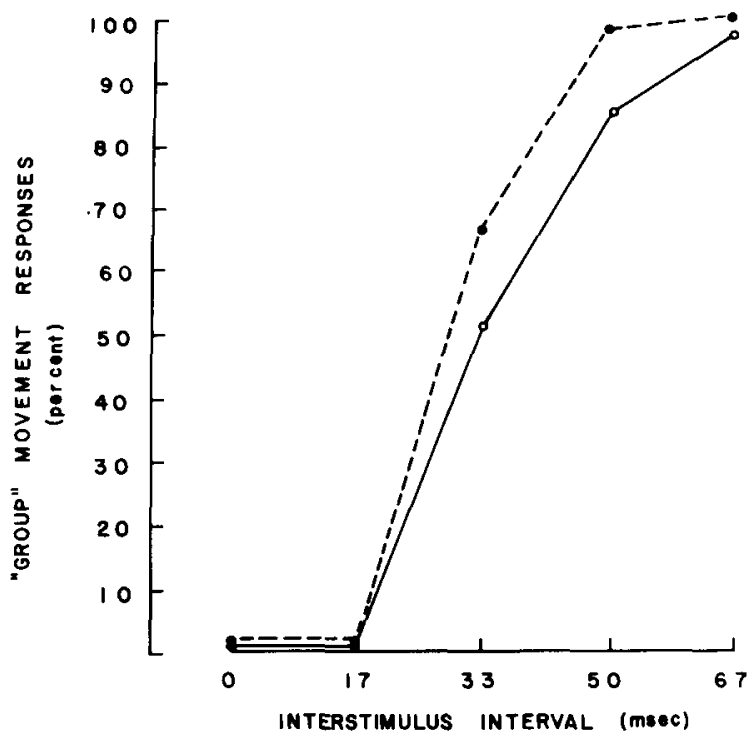

Figure 5. Percentage of group movement responses as a function of the duration of the interstimulus interval with perturbation magnitude of the end line as a parameter. Perturbation ( $Z$ ) of 0 min (control data), $\bullet$ s; and $40 \mathrm{~min}$, Os.

Position perturbation of end line. In order to determine whether (1) the changes of spatial periodicity concomitant with the perturbations of the positions of the overlapping lines or (2) a perturbation of the position of the end line ( $F$ in Figure 1$)$ would decrease the percentage of element movement sensations, another display was constructed from the two frames shown in Figure $2 \mathrm{C}$. The end-line perturbation display was like that used in the control condition (where the interelement spacing was $1 \mathrm{deg}$ ), except that the position of the end line was perturbed by making its distance from E 1 deg 40 min instead of $1 \mathrm{deg}$. The results obtained with the end-line perturbation display are given in Figure 5 along with those of the control condition. The control (dashed) and end-line perturbation (solid) curves are nearly alike. This means that the effect of introducing an aperiodicity or a change in the position of one of the end lines of one of the display frames was minimal. If anything, the end-line perturbation curve is lower than the control curve, not higher.

Interelement spacing. Figure $\mathbf{2 A}$ is a schematic representation of two stimulus frames with an interelement spacing $X$. In the present subexperiment, the computer was programmed so that, on different trials, the interelement spacing $X$ could be 1 deg (control condition), or 4,8 , or $16 \mathrm{~min}$ of visual angle. Displays with the control and three experimental spacings were used with each of five values of ISI $(0,17,33,50$, and $67 \mathrm{msec})$. Each curve in Figure 6 shows, for a single spacing $X$, the mean percentage of group movement responses as a function of ISI. 


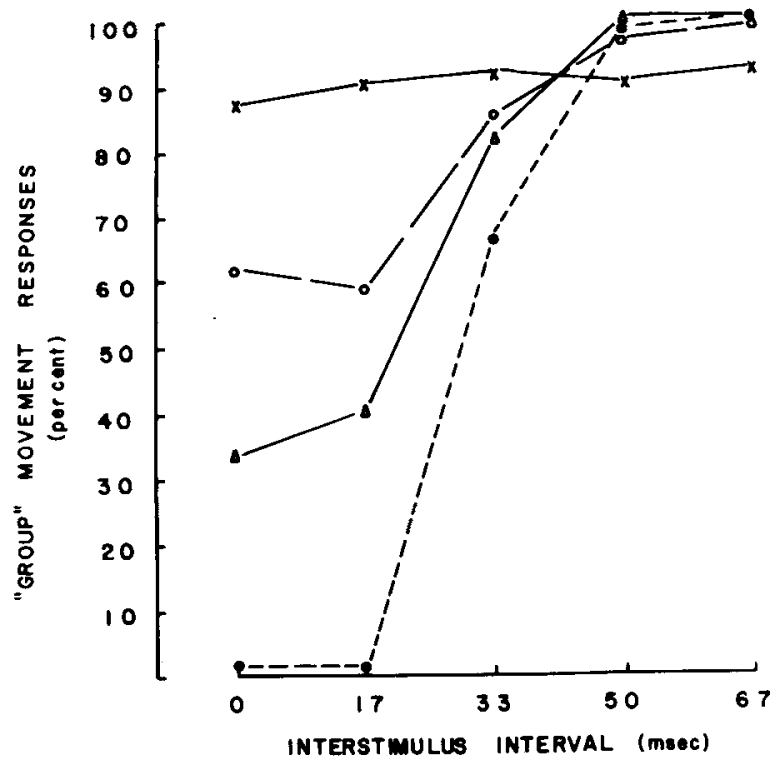

Figure 6. Percentage of group movement responses as a function of the duration of the interstimulus interval with interelement spacing as a parameter. Interelement spacing $(X)$ of 1 deg (control data), os; 16 min, $\Delta s ; 8$ min, Os; and 4 min, $x$ s.

As can be seen in Figure 6, subjects reported a greater percentage of group movement sensations with smaller spacings. In general, the curve for any smaller spacing lies above the curve for a larger spacing. As with the data for tilt and position perturbations, there was a ceiling effect at long ISIs, and the effect of interelement spacing was less pronounced there. A repeated measures analysis of variance of the percentage of group movement responses verified the significant main effect of spacing (magnitude of $X)[F(3,15)=$ $9.1, \mathrm{p}<.002]$. Also, there was a significant main effect of ISI $[F(4,20)=20.0, p<.0001]$. With the exception of the 4-min spacing, the percentage of group movement responses increased monotonically as a function of ISI for each interelement spacing.

\section{DISCUSSION}

The results of our experiments in which the orientation or position of the overlapping elements of the EG display were perturbed support the predictions advanced in the introduction, namely that such perturbations would decrease the percentage of element movement sensations. Moreover, for both tilt and position perturbations, the decrease was a direct function of the magnitude of the perturbations. The results are consistent with the idea that the perturbations disrupt the $\varepsilon$-process which links locally the individual elements of the two frames (and thereby weaken the element movement sensation) more than they disrupt the $\gamma$-process, which links the grouped elements of one frame (the three elements taken as a whole) with the grouped elements of the second frame.

One difficulty in attributing the element movement sensation entirely to a process which links elements locally is that the element at one end of one frame of the display (say A in Figure 1) must be linked with the element at the opposite end of the other frame $(F$ in Figure 1), a distance of $3 \mathrm{deg}$ and hardly a local match-up within the limit (approximately $20 \mathrm{~min}$ ) of the $\varepsilon$ - or short-range process (Braddick, 1974; Braddick \& Adlard, 1978; Petersik \& Pantle, 1979). Interestingly, a perturbation of the position of the end line did not decrease the percentage of element movement sensations. Apparently, the short-range process which mediates segregation and movement in random-dot patterns contributes to the element movement sensation only by generating a signal for the inner lines of the display, those whose points overlap or nearly overlap in successive frames. The signal generated is one of "no movement" when the points of the overlapping lines exactly correspond and one of small local movement when they nearly correspond, that is, are slightly perturbed. The signal for the movement of the end line in element movement must be generated by some other process, probably the same $\gamma$-process which accounts for the perceived displacements termed group movement. Consequently, the exact position of the end line is not critical for element or group movement.

A number of further empirical observations made with the EG display support the above interpretation of element and group movement. (1) If the overlapping elements of the typical display are presented dichoptically (i.e., elements $B$ and $C$ of one frame are presented to one eye, and elements $D$ and $E$ of the second frame to the other eye) and the end lines ( $A$ and $F$ ) binocularly, group movement is perceived almost exclusively at all ISIs. In contrast, dichoptic presentation of the end lines alone, but not the overlapping lines, does not change the proportion of element and group movement sensations from that observed when all elements are presented binocularly (Braddick \& Adlard, 1978). Presumably, local movement signals for the overlapping lines are not generated by the $\varepsilon$ - or short-range process when the overlapping lines are presented dichoptically or with a long delay between them, just as it is not possible to segregate and perceive the movement of a correlated area in random-dot patterns with dichoptic presentation or a long ISI. (2) Short ISIs that result in the perception of element movement cannot simultaneously be optimal for the generation of the local movement of the overlapping lines and for the generation of the large-amplitude movement of the end line. Short ISIs would be less than optimal for the $\gamma$-process, which is assumed to mediate the move- 
ment of the end line. Interestingly, when the display duration is extended beyond the few seconds used for a trial in our experiments, the movement of the end line in the case of element movement disappears or adapts 'within $10 \mathrm{sec}$, and the end line appears to just flash on and off in place at either end of the display. At the same time, local movement for any slightly perturbed overlapping line persists. (3) It seems safe to conclude that the process ( $\varepsilon$-process) that codes the local movement of the overlapping lines of the display is highly sensitive to spatial detail and position. The movement of the end line in the case of element movement appears to be coded by some other process akin to that which produces group movement. During group movement, the perceptual organization of the entire display is governed by a process ( $\gamma$-process) which is less sensitive to spatial detail and position, in the sense that it can tolerate small changes in the spatial structure of a stimulus that it treats as a unit. ${ }^{3}$

The effects of the manipulation of interelement spacing in our experiments remain to be explained. As the interelement spacing was decreased, observers reported a smaller percentage of element movement sensations. This result would be expected under our hypotheses because local pairings of all three elements in successive frames, rather than just the two overlapping elements, can be achieved by the $\varepsilon$-process as the interelement spacing is reduced below $20 \mathrm{~min}$. The important point to keep in mind is that what the observer calls group movement with short ISIs and a small interelement spacing may be mediated by a process (the $\varepsilon$-process) which is different from the one (the $\gamma$-process) responsible for what the observer calls group movement at long ISIs with either a small or large interelement spacing.

\section{REFERENCES}

Anstis, S. Phi movement as a substitution process. Vision Research, 1970, 10, 1411-1430.

ANSTIS, S. M. Apparent movement. In R. Held, H. W. Leibowitz, \& H.-L. Teuber (Eds.), Handbook of sensory physiology (Vol. VIII). Perception. Berlin: Springer-Verlag, 1978.

Attneave, F. Multistability in perception. Scientific American, $1971,225,62-71$.

BgLl, H., \& LAppin, J. Sufficient conditions for the discrimination of motion. Perception \& Psychophysics, 1973, 14, 45-50.

BeLL, H., \& LAPPIN, J. The detection of rotation in random-dot patterns. Perception \& Psychophysics, 1979, 26, 415-417.

BraDdick, $O$. The masking of apparent motion in random-dot patterns. Vision Research, 1973, 13, 355-369.

Braddick, O. A short-range process in apparent motion. Vision Research, 1974, 14, 519-527.

Braddick, O., \& Adlard, A. Apparent motion and the motion detector. In J. Armington, J. Krauskopf, \& B. Wooten (Eds.),
Visual psychophysics ard physiology. New York: Academic Press, $19^{7} 8$.

Julesz, B. Founriations of cy'clopean perception. Chicago: University of Chicago Press, 1971.

Pantle, A. Stroboscopic movement based upon global information in successivcly presented visual patterns. Journal of the Optical Society of America 1973, 63, 1280A.

Pantle, A., \& Piccianc, L A multistable movement display: Evidence for two separate motion systems in human vision. Science, 1976, 193, 500-502.

Petersik, J. T., \& Pantle, A. Factors controlling the competing sensations produced by a bistable stroboscopic motion display. Vision Research, 1979, 19, 143-154.

Ramachandran, V., Madhusudhan Rao, V., \& Vidyasagar, T. Apparent movement with subjective contours. Vision Research, 1973, 13, 1399-1401.

TERnUs, J. The problem of phenomenal identity. In W. D. Ellis (Ed.), A source book of Gestalt psychology. London: Routledge \& Kegan Paul, 1938.

\section{NOTES}

1. There is some disagreement between Bell and Lappin (1973, 1979) and Braddick (.974) as to whether the spatial displacement limit of the short-range process should be stated in termis of visual angle at the retina or in terms of spacing between elements within a pattern. It may be significant that the particular elements that were displaced and the dependent measures employed by Braddick were not the same as those of Bell and Lappin. Our choice of values for our independent variables reveals our prejudice for a retinal angle limit, and the results support the predictions.

2. For nonzero tilt conditicns in this experiment and for nonzero position perturbation conditions (of the "overlapping" lines) in Experiment 2, the subjects readily perceived the local movements (local rotations in Experiment 1 and local lateral shifts in Experiment 2) of the "overlapping" lines in the case of element movement. While the "overlapping" lines appeared to move locally, the third line was seen to move from one end of the display to the other across the local movement paths of the "overlapping" lines. During group movement, the change in the spatial arrangement of the group of three lines from one frame to the next for a nonzero perturbation condition easily went unnoticed unless a subject scrutinized the display sequence for such a change.

3. One final note on the evolution of our thinking about the processes which determine the alternate perceptual organizations of the EG display seems to be in order. From the beginning, we postulated two separate processes as underlying the competing sensations elicited by the EG display in much the same vein as Attneave (1971) proposed mutual inhibition between neural mechanisms as the basis of multistable phenomena. Given that the assumption of two processes was correct, we set about the task of identifying our named processes ( $\varepsilon$ - and $y$-) with hypothetical motion processes already suggested in the literature. The goal of the identification task was to avoid any unnecessary proliferation of hypothetical processes in the literature. Anstis' (1978) theoretical analysis of experiments on motion perception is much like our own. In the present paper we suggest that the $\varepsilon$-process which we identified with a short-range process in our previous papers only competes with the $\gamma$-process via its generation of local movement signals for the overlapping elements of the EG display.

(Received for publication July 9, 1979; revision accepted January 7,1980 .) 